\title{
Dual acting antidepressants: what are the key aspects in terms of short and long term clinical efficacy?
}

Stefanie Krüger

\author{
Address: Department of Psychiatry, Campus Charité-Mitte, Chariteplatz 1, 10117 Berlin, Germany \\ from WPA Thematic Conference. Coercive Treatment in Psychiatry: A Comprehensive Review \\ Dresden, Germany. 6-8 June 2007 \\ Published: 19 December 2007 \\ BMC Psychiatry 2007, 7(Suppl I):S7I doi:10.| I86/I47|-244X-7-SI-S7|
}

This abstract is available from: http://www.biomedcentral.com/I47I-244X/7/SI/S7I

(c) 2007 Krüger; licensee BioMed Central Ltd.

With treatment to remission as the gold standard for depression treatment, there is considerable reassessment of treatment approaches with the view to finding and employing agents capable of rapidly eliminating all symptoms and returning patients to normalcy. The selective serotonin reuptake inhibitors (SSRIs), the most commonly prescribed antidepressants, have a single-action mechanism involved in modulating the reuptake of the neurotransmitter serotonin. The selectivity of the SSRIs renders them safer and more tolerable than the earlier multi-acting monoamine oxidase inhibitors (MAOIs) and the tricyclic antidepressants (TCAs). However, because serotonin is not the only neurotransmitter implicated in the pathophysiology of depression, the selectivity that bestows safety to SSRIs may limit somewhat the antidepressant effect in some patients. A newer class of dualaction antidepressants is characterised by inhibiting both serotonin and noradrenaline at the presynaptic membrane and by a weak affinity with receptors at the postsynaptic membrane. These serotonin-norepinephrine reuptake inhibitors (SNRIs) have improved side effect profiles compared with the earlier multi-action antidepressants, compare favorably with the SSRIs on safety and tolerability, and reduce depression and its associated symptoms with greater rapidity. In addition, there is evidence that they may have prophylactic properties in preventing major depressive episodes. 